\title{
Diffusion Controlled Model of Opinion Dynamics
}

\author{
Anjan Kumar Chandra \\ Department of Physics, Malda College \\ Malda, West Bengal, 732101, India \\ anjanphys@gmail.com \\ Abhik Basu \\ Condensed Matter Physics Division \\ Saha Institute of Nuclear Physics \\ Kolkata 700064, India \\ abhik.basu@saha.ac.in
}

Received 25 November 2016

Accepted 5 February 2017

Published 10 March 2017

\begin{abstract}
We have studied the effect of diffusion controlled opinion dynamics on a ring lattice where agents are placed on a fraction of sites. We have chosen the diffusion on a circular ring as a simple model to study emphasizing on the fact that agents approach their nearest neighbor for exchanging opinion. The agents execute simple exclusion process (SEP) on the ring and exchange opinion with neighboring agents according to a fixed rule. Our study shows that as agent density decreases, higher conviction power is necessary to create consensus. We have also investigated the nature of active-to-absorbing state phase transition for various densities and found that there are two universality classes for density $\rho=1$ and $\rho<1$.
\end{abstract}

Keywords: Opinion; diffusion; exclusion.

\section{Introduction}

The emergence of consensus is an important issue in social science problems. ${ }^{1-4}$ The key point is that the choices of the individuals between candidates in a vote, preferred languages/culture or opinions in general are neither independent, nor random. They evolve through interactions between the individuals giving rise to an emergent consensus or a "disordered" state of co-existence. Opinion dynamics has recently attracted the attention of physicists, and there has been already some significant attempts to model such behavior in the light of rather well understood tools like phase transitions and critical phenomena. Mathematical formulations of such social

This is an Open Access article published by World Scientific Publishing Company. It is distributed under the terms of the Creative Commons Attribution 4.0 (CC-BY) License. Further distribution of this work is permitted, provided the original work is properly cited. 
behavior has helped us to understand how global consensus emerges out of individual opinions. ${ }^{5-11}$ "Opinions" are usually modeled as variables, discrete or continuous, and are subject to changes due to binary interactions, global feedback and even external factors. The main interest in these studies is to investigate the steady state properties: a state of consensus and the other with mixed beliefs.

We focus our attention to a specific class of simple model (LCCC model) proposed recently, ${ }^{12}$ having apparent similarity with kinetic models of wealth exchange. ${ }^{13}$ The opinions of individuals are continuous variables in $[-1,1]$ which change due to binary interactions. The only parameter in these models is "conviction", which is a measure of how much an individual sticks to its previous opinion while interacting with another. The system of such individuals, or the "society", reaches a state of "consensus" (ordered) if this parameter stays above a certain threshold and a transition to null opinion state below this critical value. The various critical exponents has been measured for this model and some of its variants. ${ }^{14}$ A generalized version of this model has also been studied where the fact that the ability to influence need not be identical to one's conviction has been considered. ${ }^{15}$ The discretised version of the LCCC model has also been solved exactly ${ }^{16}$ which also shows an activeabsorbing phase transition as was seen in the continuous version and the idea of bounded confidence has also been implemented. ${ }^{17}$ The percolation transition has also been studied on this model in two dimension. ${ }^{18}$

In most real life situations, agents (e.g., human beings) not only exchange opinion, but are also mobile - they move from one place to another, leading to generation of effective interactions due to correlations of the density fluctuations and the associated slow dynamics of densities due to the conservation law of mass. Conservation laws are known to affect universal scaling properties at the phase transitions; compare, for instance, the difference between the dynamic exponents for models A and B (in the nomenclature of Ref. 19). Thus, the role of agent density fluctuations should be of importance in opinion spreading and its associated phase transitions. For a particular value of conviction, it is quite obvious that in less populated regions, it will take much longer time to reach the state of consensus. In this paper, we study the LCCC model on a one-dimensional (1D) ring, where agents are placed on a fraction of sites and the agents can hop to vacant sites at a predetermined rate. With all sites filled, we retrieve the results of the original LCCC model. In this paper, we first discuss the model, we study and then in the next section we study the phase transition behavior of the model using non-equilibrium relaxation (NER) and the corresponding critical exponents.

\section{The Model}

In our model, the agents are located on a $1 \mathrm{D}$ ring of $N$ sites with density $\rho$. So total number of agents are $\rho N$. Now at a time $t$, we select a site $i$ randomly. We define two dynamical variables at each site: the density $\rho_{i}$ and its opinion $o_{i}$. While $\rho_{i}$ is discrete and can be 0 (empty site) or 1 (occupied), opinion $o_{i}$ is a number bounded between 


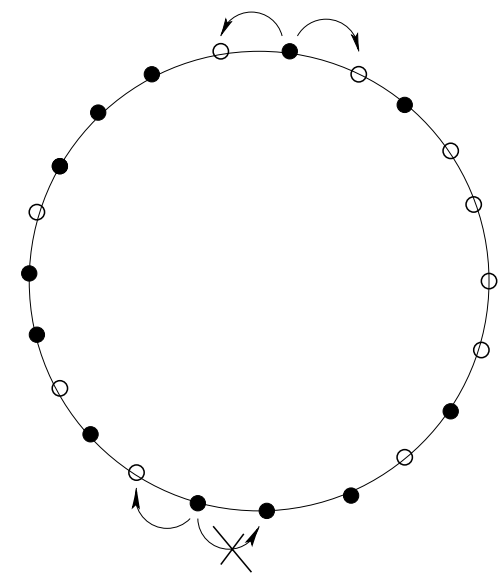

Fig. 1. Ring consisting of $N$ sites, with $\rho N$ agents placed on the blackened sites and the white circles are vacant.

$-1 \leq o_{i} \leq+1$ for all $i$. Further, $o_{i}$ is "tagged" to $\rho_{i}$, i.e., $o_{i}$ is non-zero only if $\rho_{i}$ is non-zero $(=1)$, when an agent exists. Density $\rho_{i}(t)$ executes symmetric exclusion process ((SEP); equivalently, 1D diffusion) along the lattice, in which an individual particle can jump to its right and left nearest neighbor sites which are vacant with equal probability. Since every $o_{i}$ is tagged to $\rho_{i}$, SEP for $\rho_{i}$ implies SEP for $o_{i}$ as well. If two neighboring sites are occupied, there is no SEP taking place between them. Instead, the two adjacent agents may exchange opinion according to the following rule: if an agent is present at that site with opinion $o_{i}(t)$, we select a nearest neighbor $j$ (left/right) with a probability $1 / 2$. In this model, also the opinions are bounded by $-1 \leq o_{i} \leq+1$ for all $i$. Now if an agent is present at the selected neighboring site $j$ with opinion $o_{j}(t), j=i-1, i+1$, then the two agents exchange opinions between each other by binary interactions as follows:

$$
\begin{aligned}
& o_{i}(t+1)=\lambda\left[o_{i}(t)+\epsilon o_{j}(t)\right], \\
& o_{j}(t+1)=\lambda\left[o_{j}(t)+\epsilon^{\prime} o_{i}(t)\right],
\end{aligned}
$$

where $\epsilon, \epsilon^{\prime}$ are drawn randomly from uniform distributions in $[0,1]$. Here, $\lambda$ is the conviction parameter that determines the extent up to which an individual retains its own opinion. Thus, the overall dynamical rules for $\rho_{i}$ and $o_{i}$ are given by

$$
\begin{aligned}
& \rho_{i}(t+1)=\left[\rho_{i+1}(t)+\rho_{i-1}(t)-2 \rho_{i}(t)\right] / 2, \\
& o_{i}(t+1)= \lambda \rho_{i} \rho_{i+1}\left[o_{i}(t)+\epsilon_{i+1}(t) o_{i+1}(t)\right] / 4 \\
&+\lambda \rho_{i} \rho_{i-1}\left[o_{i}(t)+\epsilon_{i-1}(t) o_{i-1}(t)\right] / 4 \\
&+\left[o_{i-1}(t) \rho_{i-1}\left(1-\rho_{i}\right)+o_{i+1}(t) \rho_{i+1}\left(1-\rho_{i}\right)\right. \\
&\left.+o_{i}(t) \rho_{i}\left(1-\rho_{i+1}\right)+o_{i}(t) \rho_{i}\left(1-\rho_{i-1}\right)\right] / 2 .
\end{aligned}
$$

Evidently, the update rule (3) for $o_{i}$ reduces to those in Ref. 12 in the fully filled limit, i.e., $\rho_{i}=1$ for all $i$. 
The ordering in the system is measured by the parameter $O=\left|\sum_{i} o_{i}\right| / \rho N$, which denotes the average opinion. If there is no agent present at the selected neighboring site, then the agent at site $i$ simply moves to the neighboring site with a rate unity. The system equilibrates to two possible phases. Below a critical value $\lambda_{c}, o_{i}=0$ for all agents. Whereas for $\lambda>\lambda_{c}, O>0$. The critical value of $\lambda$ depends on the concentration of agents. As $\lambda$ goes to $1, O \rightarrow 1$. So the system shows critical behavior at $\lambda_{c}$ characterized by the critical exponents. We have studied the critical values of the exchange parameter $\lambda$ for different densities and how the critical exponents change with $\rho$. The maximum value of $\rho=1.0$ yields the original LCCC model.

\section{Results}

To study the opinion behavior in LCCC model for lower agent densities, we have performed extensive Monte-Carlo simulations of the model update rules (2) and (3) by random updates. We study the system for two values of the average density $\rho=0.50$ and $\rho=0.10$. Unsurprisingly, our results for $\rho=1.0$ fully agree with the original LCCC model. ${ }^{12}$ Just as for $\rho=1.0$, with other densities $\rho=0.5,0.1$ as well our results show an active steady state with a non-zero value of the order parameter $O$ for sufficiently high $\lambda$ and an absorbing state with $O=0$ for sufficiently low $\lambda$. This suggests a generic active-to-absorbing state phase transition (AAPT) with $\lambda$ as the control parameter in our model for arbitrary densities. This calls for more detailed investigations concerning the nature of AAPT and its possible $\rho$-dependences. Our investigations reveal second-order AAPTs. In order to analyze the AAPT systematically, we have carefully measured the transition point and a set of scaling exponents for $\rho=0.5,0.1$.

We have measured the critical value of $\lambda$ and the critical exponents for two typical values of $\rho=0.10$ and 0.50 . It was found that the critical value $\lambda_{c}$ increases slightly with decreasing density. The value of $\lambda_{c}$ is 0.66687 for $\rho=0.50$ and 0.66730 for $\rho=0.10$. It suggests that as density of agents decreases it needs higher conviction power to achieve consensus.

The order parameter exponent $\beta$ is defined as:

$$
O \sim\left(\lambda-\lambda_{c}\right)^{\beta} \text {. }
$$

Although $\rho=1.0$ yields no clear unambiguous scaling for the order parameter, for both $\rho=0.50$ and 0.10 , the value of $\beta$ is $1.15 \pm 0.01$ (Fig. 2).

At the critical point, the order parameter will relax asymptotically following a power law of the form $O \sim t^{-\delta}$. The value of $\delta$ is $0.95 \pm 0.01$ for $\rho=0.50$ and 0.10 (Fig. 3), whereas it is $1.00 \pm 0.05$ for $\rho=1.0$. This suggests that for density lower than unity the order parameter relaxation is slower.

We have also studied the relaxation time of the order parameter. The order parameter $O$ relaxes to equilibrium in time $t$ as,

$$
O(t) \propto \exp (-t / \tau)
$$




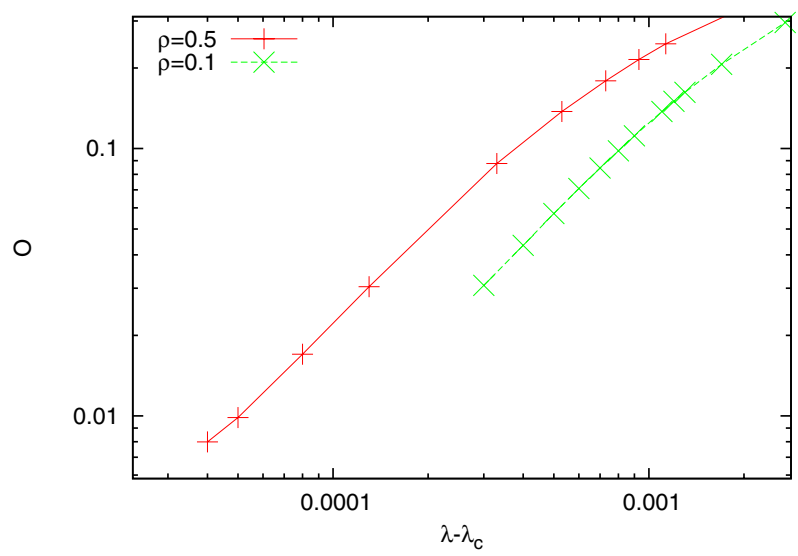

Fig. 2. Variation of average opinion with $\lambda$ near critical points for different values of $\rho$. The value of $\beta$ is $1.15 \pm 0.01$.

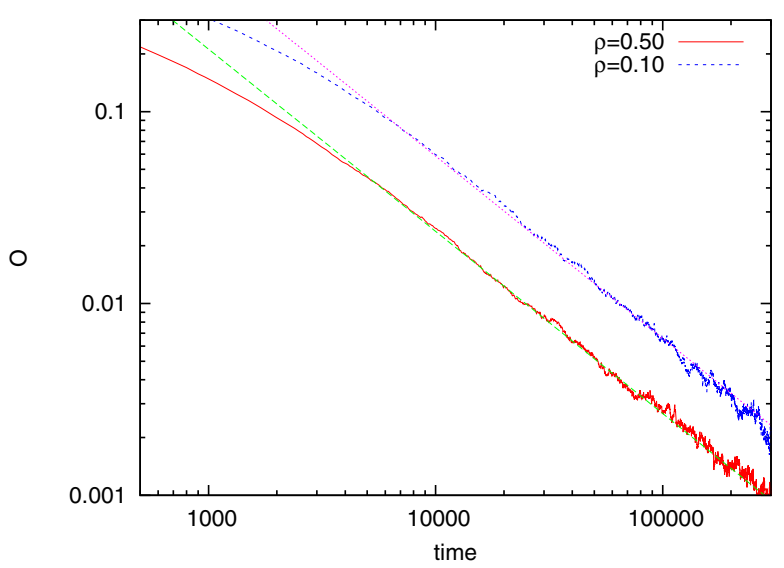

Fig. 3. Variation of average opinion with time at critical points for $\rho=0.50$ and $\rho=0.10$. The value of $\delta$ is $0.95 \pm 0.01$ for both $\rho=0.50$ and $\rho=0.10$.

for the symmetric phase $\left(\lambda<\lambda_{c}\right)$ and

$$
O(t) \propto O_{\text {eq }}[1-\exp (-t / \tau)]
$$

in the symmetry-broken phase $\left(\lambda>\lambda_{c}\right), O_{\text {eq }}$ being the equilibrium value of $O$ and $\tau$ is the relaxation time. From the relaxation behavior of $O$ for different values of $\lambda$ away from the critical point, we estimate the value of $\tau$. The relaxation time diverges algebraically as

$$
\tau \sim\left|\lambda-\lambda_{c}\right|^{-z}
$$

where $z$ is the relaxation exponent. The value of the exponent is $1.25 \pm 0.02$ for $\rho=0.10$ and $\rho=0.50$ (Fig. 4), which are same up to the error limit, but drastically different from the value of $z=0.97 \pm 0.01$ for $\rho=1.0$. 


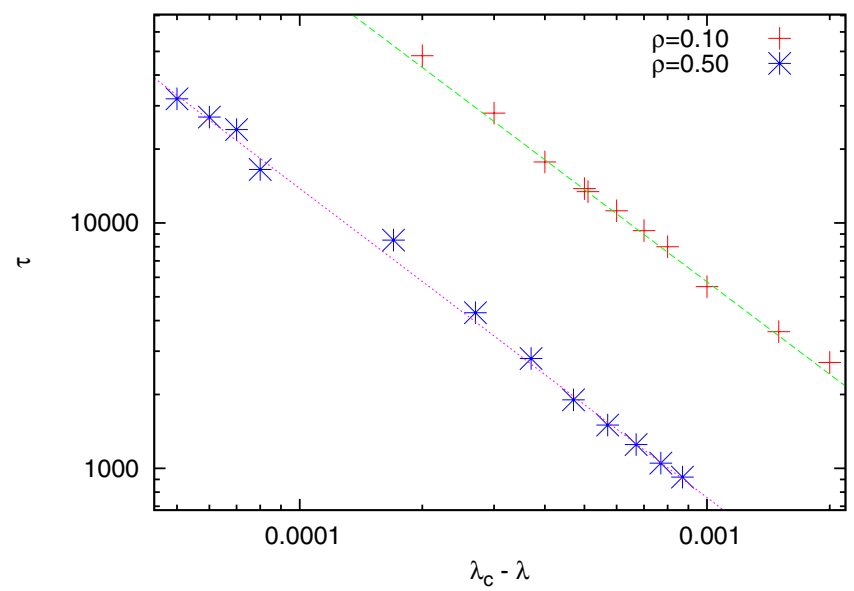

Fig. 4. Variation of relaxation time with $\lambda$ at critical points for different values of $\rho=0.50$ and $\rho=0.10$, the value of $z$ is $1.25 \pm 0.01$ for both values of $\rho$.

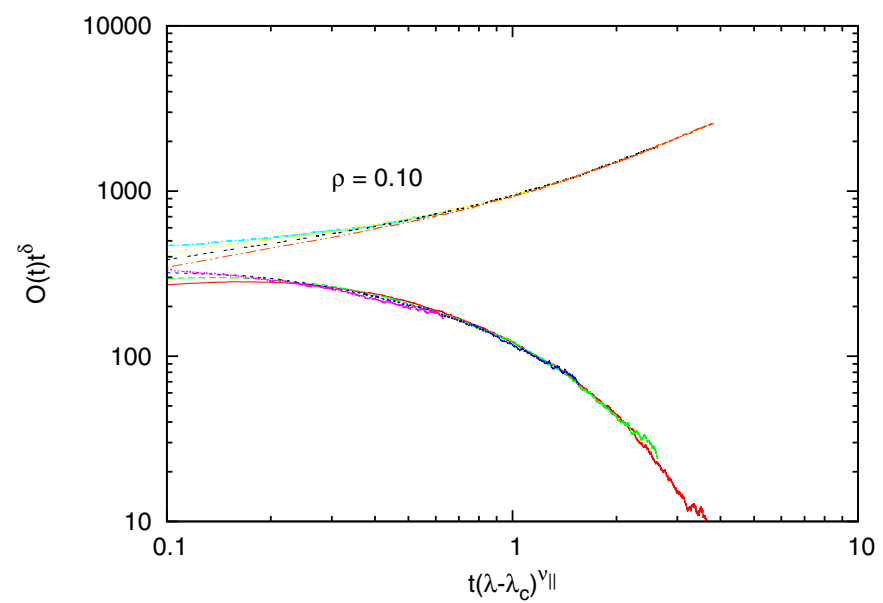

Fig. 5. Variation of average opinion with time at critical points for $\rho=0.10$. The value of $\nu_{\|} \approx 1.40 \pm 0.05$.

From finite size scaling theory, ${ }^{20}$ it is known that the order parameter $O$ follow a scaling relation of the form

$$
O(t) \approx t^{-\delta} \mathcal{F}\left(t^{1 / \nu_{\|}}\left(\lambda-\lambda_{c}\right)\right),
$$

where $\mathcal{F}$ is a universal scaling function of a form such that for large argument, the time dependence vanishes. From the data-collapse of the off-critical relaxation curves, we obtain the correlation time exponent to be $\nu_{\|} \approx 1.30 \pm 0.05$ for $\rho=0.10$ (Fig. 5) and $\nu_{\|} \approx 1.40 \pm 0.05$ for $\rho=0.50$ (Fig. 6), which is again different from $\rho=1.0$.

Below we present the various critical exponents for $\rho=1$ and $\rho<1.0$ in a tabular form (Table 1). 


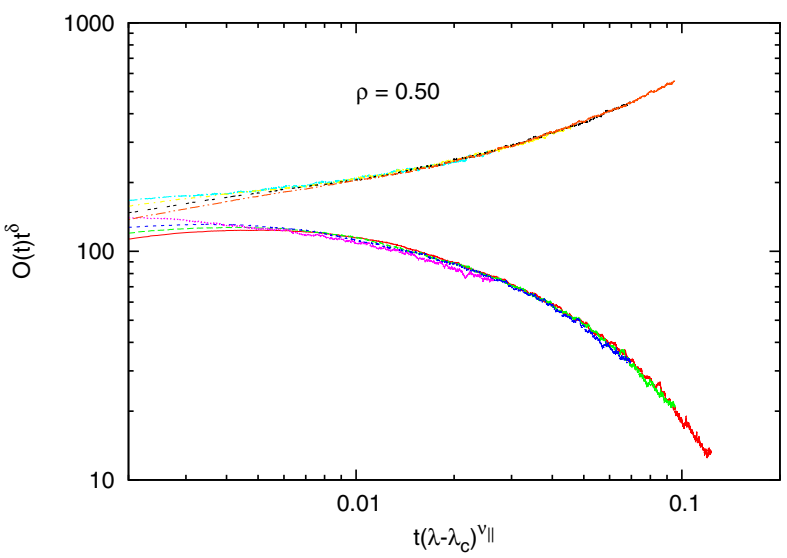

Fig. 6. Variation of average opinion with time at critical points for $\rho=0.50$. The value of $\nu_{\|} \approx 1.40 \pm 0.05$.

Table 1. Comparing the different exponents for different densities.

\begin{tabular}{ccccc}
\hline$\rho$ & $\beta$ & $z$ & $\delta$ & $\nu_{\|}$ \\
\hline 1.00 & $0.10(1)$ & $0.97(1)$ & $1.00(5)$ & $1.20(1)$ \\
0.50 & $1.15(1)$ & $1.25(2)$ & $0.95(5)$ & $1.40(5)$ \\
0.10 & $1.15(1)$ & $1.25(2)$ & $0.95(5)$ & $1.40(5)$ \\
\hline
\end{tabular}

We thus find that the critical exponents are substantially different for $\rho=0.10,0.50$ from their values in the fully filled limit $(\rho=1)$, as reported here (see also Refs. 12 and 14 for results with $\rho=1.0$ ). Furthermore, the critical exponents for $\rho=0.1,0.5$ are found practically to be the same (within error bars). This suggests that there are indeed two different universality classes in the model with $\rho=1.0$ and $\rho<1.0$. The fact that the scaling properties at the AAPT change for $\rho<1$, can be understood qualitatively as follows. When $\rho<1$, all the sites are not filled and there are local diffusive density fluctuations. Due to the conservation of mass, these density fluctuations execute slow dynamics. Since the dynamics of the local opinion $o_{i}$ (i.e., the update rules 3) depend on $\rho_{i}$, any fluctuations in $\rho_{i}$ should appear as relevant multiplicative noises in the dynamics of $o_{i}$. It is reasonable to expect that these multiplicative noises affect the critical scaling behavior at the AAPT. Further, density fluctuations satisfy the same diffusion equation (i.e., the same update rules 2) for all $\rho<1$. Thus, density fluctuations do not depend on the value of $\rho<1$. This is consistent with our results that the critical exponents for $\rho=0.1,0.5$ are within error bars.

\section{Conclusion}

This paper is a part of the special issue to be published on socio-econo physics. Diffusion of information plays a very crucial role in the modern world. But in remote 
rural areas where people are not equipped with modern devices, information is diffused by talking with others, sending written messages, distributing pamphlets, announcements through microphones etc. From that perspective, our study discloses the nature of opinion dynamics where opinion is spread through diffusion of agents which act as information carrier. Our study shows that as agent density decreases, higher conviction power is necessary to achieve consensus. The approach towards consensus becomes slower but the divergence of relaxation time at critical conviction becomes faster. But quantitatively, the exponents remain same for $\rho=0.1$ and 0.5. Although we have limited our study on a ring lattice and also the mobility of agents has been restricted to a single step, this study can be extended to higher dimensional lattice and also the mobility may be increased by allowing larger movements of the agents. Moreover, the effect of diffusion may also be studied for the case that involves only discrete opinions.

\section{Acknowledgments}

Anjan Kumar Chandra gratefully acknowledges the financial support from Science and Engineering Research Board (SERB), DST, Government of India [Project file No. ECR/2016/000848]. Abhik Basu gratefully acknowledges partial financial support from Alexander von Humboldt Stiftung, Germany under the Research Group Linkage Programme (2016).

\section{References}

1. B. K. Chakrabarti, A. Chakraborti and A. Chatterjee (eds.), Econophysics and Sociophysics (Wiley-VCH, Berlin, 2006).

2. D. Stauffer, Encyclopedia of Complexity and Systems Science, (ed.) R. A. Meyers (Springer, New York, 2009); Comp. Sci. Eng. 5 (2003) 71.

3. C. Castellano, S. Fortunato and V. Loreto, Rev. Mod. Phys. 81 (2009) 591.

4. S. Galam, Y. Gefen and Y. Shapir, J. Math. Sociology 9 (1982) 1.

5. T. M. Liggett, Interacting Particle Systems: Contact, Voter and Exclusion Processes (Springer-Verlag, Berlin, 1999).

6. K. Sznajd-Weron and J. Sznajd, Int. J. Mod. Phys. C 11 (2000) 1157.

7. S. Galam, Int. J. Mod. Phys. C 19 (2008) 409.

8. R. Hegselman and U. Krause, J. Art. Soc. Soc. Simul. 5 (2002) 2.

9. G. Deffuant, N. Neau, F. Amblard and G. Weiss, Adv. Complex Syst. 3 (2000) 87.

10. S. Fortunato, Int. J. Mod. Phys. C 16 (2005) 17.

11. G. Toscani, Commun. Math. Sci. 4 (2006) 481.

12. M. Lallouache, A. S. Chakrabarti, A. Chakraborti and B. K. Chakrabarti, Phys. Rev. E 82 (2010) 056112.

13. A. Chakraborti and B. K. Chakrabarti, Eur. Phys. J. B 17 (2000) 167; A. Chatterjee, B. K. Chakrabarti and S. S. Manna, Physica A 335 (2004) 155; A. Chatterjee and B. K. Chakrabarti, Eur. Phys. J. B 60 (2007) 135.

14. S. Biswas, A. K. Chandra, A. Chatterjee and B. K. Chakrabarti, J. Phys., Conf. Ser. 297 (2011) 012004.

15. P. Sen, Phys. Rev. E 83 (2011) 016108.

16. S. Biswas, Phys. Rev. E 84 (2011) 056106. 
17. P. Sen, Phys. Rev. E 84 (2012) 016115.

18. A. K. Chandra, Phys. Rev. E 85 (2012) 021149.

19. P. Hohenberg and B. I. Halperin, Rev. Mod. Phys. 49 (1977) 435.

20. H. Hinrichsen, Adv. Phys. 49 (2000) 815. 